\title{
Avaliação de periódicos eletrônicos acadêmicos brasileiros: uma proposta de método baseado na análise de links para o site do periódico'
}

\author{
Evaluation of Brazilian academic electronic journals: \\ a proposal for a methodology based on an analysis \\ of links to the journal website
}

Carlos Henrique MARCONDES²

Marília Alvarenga Rocha MENDONÇA ${ }^{3}$

RESUMO

Este artigo é resultado de projeto de pesquisa patrocinada pelo Conselho Nacional de Desenvolvimento Científico e Tecnológico, com a finalidade de desenvolver um método de avaliação para periódicos eletrônicos brasileiros em ciência e tecnologia, considerados emergentes pela pesquisa. Ó método se propõe a ser uma alternativa ao fator de impacto utilizado pelo Institute for Scientific Information; é baseado na análise quantitativa e qualitativa de links efetuados para o site de um periódico eletrônico, obtidos por meio da submissão de sua URL ao site de busca Google. Considerou-se não somente a quantidade de links direcionados ao site, mas também a existência de sites considerados autoridades na área do periódico pesquisado e links de instituições estrangeiras feitos para o site do periódico. $\mathrm{O}$ artigo propõe uma fórmula para calcular o grau de relevância do periódico analisado, atribuindo pesos a diferentes tipos de links. Os resultados obtidos foram validados por meio da opinião de especialistas de diferentes áreas do conhecimento.

Palavras-chave: comunicação científica; publicações eletrônicas; periódicos eletrônicos; links; avaliação; Brasil.

\section{A B S TRACT}

This paper reports the results of a research project, sponsored by Conselho Nacional de Desenvolvimento Científico e Tecnológico - in order to develop an evaluation methodology for emerging Brazilian e-journals on Science and Technology. The proposed methodology intends to be an alternative for the impact factor used by the Institute for Scientific Information, and is based on the quantitative and qualitative analysis of the links to the e-journal website, obtained by submitting its URL to the Google search engine. The methodology considers

\footnotetext{
1 Revisão e ampliação de trabalho originalmente apresentado no 8th ELPUB - International Conference on Electronic Publishing, Brasília, June, 2004 e no SNBU - Seminário Nacional de Bibliotecas Universitárias, Natal, out. 2004.

${ }_{2}$ Professor, Instituto de Artes e Comunicação Social, Departamento de Documentação, Universidade Federal Fluminense. Rua Prof. Lara Vilela, 126, São Domingos, 24210-590, Niterói, RJ, Brasil. Correspondência para/Correspondence to: C.H. MARCONDES. E-mail: <marcon@vm.uff.br>.

${ }^{3}$ Professora, Instituto de Artes e Comunicação Social, Departamento de Documentação, Universidade Federal Fluminense. Niterói, RJ, Brasil.

Esta pesquisa contou com a participação de Ana Carolina de Araújo Nogueira e Cláudia Maria Carvalho, alunas do Curso de Biblioteconomia e Documentação, Universidade Federal Fluminense, Bolsistas PIBIC/CNPq/UFF.

Recebido em 11/11/2005 e aceito para publicação em 4/5/2006.
} 
not only the number of links directed to the website, but also the existence of qualitative links: websites which are considered an "authority" on specific knowledge areas, and those of non-Brazilian institutions. These different kinds of links were weighted and used to calculate a grade to an e-journal site, as an indicator of its relevance in a certain knowledge area. The results obtained were validated by specialists in different knowledge areas.

Key words: scientific communication; electronic publishing; electronic journals; links; evaluation; Brazil.

\section{N T RO D U Ç Ã O}

Periódicos eletrônicos constituem uma recente realidade no cenário da Web brasileira. Encontra-se em fase final de desenvolvimento, um projeto de pesquisa patrocinada pelo Conselho Nacional de Desenvolvimento Científico e Tecnológico (CNPq), cujo objetivo é conhecer a situação dos periódicos eletrônicos brasileiros em ciência e tecnologia (MARCONDES et al., 2004). Foram encontrados cerca de 500 periódicos eletrônicos, em diferentes áreas do conhecimento; aproximadamente 400 são periódicos impressos que possuem, também, uma versão eletrônica, e 89 são publicados apenas eletronicamente. O universo desta pesquisa é constituído pelos 89 periódicos científicos que apresentam apenas a versão eletrônica.

O universo dos periódicos eletrônicos brasileiros apresenta diferentes níveis de qualidade. Para os periódicos acadêmicos existem políticas públicas emanadas de agências brasileiras vinculadas ao desenvolvimento científico e tecnológico. No caso específico dos periódicos eletrônicos, existe um projeto patrocinado por várias agências governamentais denominado - Scientific Electronic Library Online (SciELO) (PACKER, 2000), destinado a desenvolver um portal na WEB, oferecendo a versão eletrônica dos mais importantes periódicos científicos brasileiros e exigindo rígidos critérios de qualidade para os periódicos que o integram.

No cenário brasileiro dos periódicos eletrônicos, o portal SciELO cumpre um papel de destaque. Produto de uma parceria entre diversas instituições, nacionais e internacionais: (Fundação de Amparo à Pesquisa do Estado de São Paulo (FAPESP) - <http:// www.fapesp.br>, Centro de Informação em Ciências da Saúde para a América Latina e o Caribe (BIREME) - <http://www.bireme.br>, uma organização pertencente a Organização Panamericana de Saúde (PAHO) - e a Organização Mundial de Saúde (WHO), todas elas relacionadas à comunicação científica, juntamente com vários editores de publica- ções científicas. Atualmente, o portal SciELO é considerado um importante componente das políticas públicas brasileiras no que diz respeito ao desenvolvimento da ciência, disseminando, no mundo todo, a literatura técnico-científica publicada nos países em desenvolvimento e aumentando, assim, sua visibilidade muito além de suas fronteiras.

O projeto SciELO/Brasil teve início em 1998, com a migração, para versão eletrônica, de um certo número de periódicos brasileiros impressos considerados de alta qualidade, pertencentes às áreas de Ciências da Saúde e Biologia. Hoje existem outros portais SciELO, contendo periódicos eletrônicos de países da América Latina e do Caribe. O portal SciELO disponibiliza os mais importantes periódicos científicos brasileiros, sendo a maioria deles periódicos acadêmicos publicados em formato papel. Por meio da pesquisa citada verificou-se que, além dos periódicos eletrônicos incluídos no SciELO, existem, aproximadamente, 89 periódicos eletrônicos referentes a diferentes áreas do conhecimento, que se convencionou chamar de periódicos eletrônicos emergentes. Diferentemente daqueles arrolados pelo SciELO, esses periódicos são recentes, apresentam uma periodicidade irregular, não se constituindo, portanto, publicações sólidas. Falta a tais periódicos um método de avaliação sistemático e consistente (PACKER, 2001).

Acredita-se que uma das barreiras para o uso sistemático desses periódicos ditos emergentes pela comunidade científica brasileira seja a ausência de uma avaliação qualitativa. São conhecidas as dificuldades para que periódicos de países do Terceiro Mundo tenham maior visibilidade (GIBBS, 1995; GINSPARG, 1996; TARGINO; GARCIA, 2000). Esses periódicos apresentam inúmeras dificuldades em atender aos critérios do Institute for Scientific Information (ISI) (TESTA, 1997). Por outro lado, por muitas vezes refletirem visões e problemas locais que geralmente não despertam interesse nas bases de dados e publicações do Primeiro Mundo, esses periódicos devem ser incentivados. 
Uma biblioteca universitária, ao assinalar na sua home page um link para o site de um periódico eletrônico não está recomendando esse periódico? Por isso, esta pesquisa pretende propor e delinear um método de avaliação para ser utilizado nos periódicos eletrônicos brasileiros em ciência e tecnologia, ditos emergentes, alternativos ao fator de impacto utilizado pelo ISI, contemplando uma análise quantitativa e qualitativa dos links feitos ao site do periódico.

Desde o aparecimento do $1^{\circ}$ periódico científico no século XVII, o Journal des Sçavans, e do Philosophical Translations of the Royal Society, em 1665 (DAY, 1999; MEADOWS, 1999), os periódicos científicos são considerados e institucionalizados pela comunidade científica como meio de disseminação dos resultados de suas pesquisas, assegurando prioridade e qualidade, além de prover e preservar o conhecimento científico.

A World Wide Web (WEB) vem crescendo expressivamente nos últimos anos, tornando-se um novo meio de comunicação e um grande repositório de informações, promovendo um crescimento na economia mundial do conhecimento. Cientistas e estudiosos vêem a Web como um meio mais visível (LAWRENCE, 2001), rápido e barato para publicação de suas pesquisas, em comparação com os tradicionais periódicos impressos (ODLYSKO, 1998). É constituída por documentos eletrônicos, páginas lincadas, isto é, conteúdos inter-relacionados: uma página exibida na tela de um navegador traz um link que permite navegar para outras páginas, constituindo a assim chamada teia global de bilhões de páginas interconectadas.

Kleinberg e Lawrence (2001) afirmam que devido ao crescimento não planejado da Web, ela tem sido considerada sem estrutura. Entretanto, pesquisas recentes já consideram a WEB uma rede auto-organizada. A base para sua auto-organização seria sua estrutura de links. O estudo de estrutura de links da WEB é conhecido como análise de links. A pesquisa sobre análise de links encontrou interessantes insigts na área de desenvolvimento de programas crawlers, spiders e robots. Kleimberg (1998) encontrou dois tipos de sites, baseando-se nos estudos sobre links de e para um conjunto de sites: autoridades, sites que são lincados de muitas outras páginas, e hubs, páginas que lincam para muitas páginas.

$\mathrm{Na}$ área da Ciência da Informação, considerando o contexto da comunicação científica e da
Biblioteconomia, citação de um periódico científico para outro consiste em um conhecido mecanismo de avaliação da relevância de determinado autor. Esse mecanismo indica uma recomendação que se faz de um autor para outro e, indiretamente, a recomendação para o periódico científico que publica o artigo. Essa sistemática de citação consiste na base de cálculo de fator de impacto (GARFIELD, 1994), reconhecido indicador da relevância de publicações científicas. $O$ ISI, fundado por Eugene Garfield, é uma instituição que coleta informações acerca de publicações científicas em todo o mundo, realizando análises estatísticas dos dados obtidos e calculando o fator de impacto de milhares de periódicos científicos. $O$ fator de impacto consiste, então, em um valioso indicador para avaliação de publicações científicas, que auxilia no planejamento de políticas científicas e de políticas específicas para bibliotecas, como, por exemplo, no desenvolvimento de acervo.

Com o surgimento da Web, a pesquisa em Ciência da Informação rapidamente fez uma analogia entre citações e links. Correlatos à problemática enfocada nesta pesquisa são trabalhos como o de Snyder e Rosenbaun (1999), que analisam a adequação do uso de mecanismos de busca como ferramentas para análise de links, e de Vaughan e Thewall (2003), que analisam a evolução desses mecanismos a partir da proposta do Google de usar links para um site como indicador de popularidade e, especificamente, o uso de links para sites de periódicos acadêmicos. Dessa maneira, um link pode não ser apenas um auxílio para a navegação, permitindo ao leitor, por exemplo, navegar até o fim da página, voltar à home page etc. Como afirma Vaughan e Hysen (2002, p.356), "The undelying assuption of link populariry is that the number of links to a Web site indicates the importance or quality of the site and that Web links are analogous to academic citations". Assim, o criador de uma página A, ao assinalar nela um link para a página $B$, está indicando que considera a página $B$, de alguma maneira, relacionada semanticamente à página $A$.

Um link de uma página para outra, como acontece nos estudos bibliométricos, é considerado análogo a uma citação. A hipótese com a qual se trabalhou na pesquisa, considerando-se o significado de uma citação, difere um pouco do significado de uma citação segundo os estudos bibliométricos. Diferentemente dos links para um artigo de periódico, 
links para o site de um periódico eletrônico são, geralmente, feitos pelos serviços de referência eletrônica, um serviço emergente oferecido, geralmente, pelas bibliotecas acadêmicas ou universitárias. Serviços de referência eletrônica sinalizam a existência de um processo de avaliação dos links incluídos no serviço. Dessa forma, este projeto trabalha com a seguinte hipótese: links para o site de um periódico eletrônico, levando-se em consideração o site que faz o link, significa uma recomendação para o periódico. A natureza da instituição que lincou para o periódico eletrônico pode representar um forte indicador de sua relevância.

Nas seções seguintes é apresentado o método de análise, e são apresentados e discutidos seus resultados. Na seção final são apresentados os conclusões e os futuros desenvolvimentos possíveis para esta pesquisa.

\section{MÉ TODO}

O método adotado na pesquisa considera os links direcionados ao site de um periódico eletrônico e realiza uma análise quantitativa e qualitativa dos mesmos. Os links para os sites dos periódicos eletrônicos analisados foram obtidos submetendo suas URL ao mecanismo de busca do Google, sendo consideradas somente as dez primeiras páginas resultantes. Quando um grupo de periódicos eletrônicos pertencentes a uma área específica do conhecimento era analisado, os resultados da pesquisa no Google eram colhidos em um mesmo dia. A fórmula utilizada para pontuar os periódicos analisados considera três tipos de links: o total de links (não considera os links repetidos), os links oriundos de sites estrangeiros (pertencentes a sites fora do Brasil) e os links autoridades, cada um deles recebendo um peso diferente.

Os sites autoridades (links autoridades) precisam ser, na realidade, aprovados pelas comunidades de pesquisa e pelas bibliotecas, considerando as específicas áreas do conhecimento. Nesta pesquisa, consideraram-se links autoridades os sites de bibliotecas universitárias com programas de pósgraduação, os sites de programas de pós-graduação, os sites de associações científicas e os sites de associações profissionais, considerando, em todos os casos, as respectivas áreas de atuação.
A fórmula criada para pontuar os periódicos eletrônicos considera o total de links efetuado para o site do periódico eletrônico. Desse total são excluídos os links originários do próprio site do periódico; assim, se o periódico está incluído no portal SciELO, os links para o site do periódico eletrônico vindos de alguma página do site do SciELO não são considerados, pois existem diferentes páginas no site do SciELO lincando para um periódico eletrônico e mostrando o escopo do periódico, estatísticas de acesso, fator de impacto etc., em português, espanhol e inglês. Em periódicos não incluídos no SciELO, os links oriundos do próprio site do periódico eletrônico também não são considerados.

Do total de links encontrados subtrai-se o total de links estrangeiros e o total de links autoridades, pois esses serão considerados com peso diferente. O resultado encontrado é considerado o total bruto de links, que é adicionado ao total de links estrangeiros, ao qual é atribuído peso 2, e adicionado ao total de links autoridades, ao qual é atribuído peso 3. O resultado final dessa operação resulta na pontuação atribuída ao periódico eletrônico. A fórmula utilizada é a seguinte:

$T I$ = total de links para o site do periódico eletrônico, encontrado através do Google;

$T s=$ total de links oriundos do site do SciELO ou o total de links oriundos do site do próprio periódico eletrônico;

$\operatorname{Tr}=$ total de links repetidos;

$T e=$ total de links estrangeiros;

$\mathrm{Ta}=$ total de links autoridades;

$G$ = fórmula calculada para o periódico eletrônico pontos;

Sendo $G$ calculada da seguinte forma:

$$
\mathrm{G}=3 \times \mathrm{Ta}+2 \times \mathrm{Te}+1 \times(\mathrm{TI}-\mathrm{Ts}-\mathrm{Tr}-\mathrm{Te}-\mathrm{Ta})
$$

Depois de aplicada a fórmula proposta, procurou-se confirmar, através de consulta a especialista da área de cada periódico analisado, o resultado obtido. Esse método foi aplicado, primeiramente, na área de conhecimento da Ciência da Informação, por ser essa uma área familiar ao grupo de pesquisa. Após essa experiência, o método foi aplicado em periódicos eletrônicos brasileiros de outras áreas do conhecimento, sempre em grupos de dois ou três para que fosse possível comparar os fatores e os pesos usados no cálculo de suas pontuações. As áreas do conhecimento de acordo 
com a classificação da CAPES/CNPq pesquisadas foram: Ciências da Saúde - Enfermagem (2 periódicos) e Saúde Pública (3 periódicos); Humanidades - Educação (2 periódicos); Ciências Sociais Aplicadas - Ciência da Informação e Administração (3 periódicos de cada); Ciências Agrárias - Agronomia e Veterinária (3 periódicos). Uma dificuldade adicional na pesquisa foi encontrar, entre os periódicos emergentes levantados pela pesquisa citada (89 periódicos), grupos de 2 a 3 periódicos da mesma área que fossem do mesmo escopo e pudessem ser comparados quanto a sua relevância. Por esse motivo, o método proposto só pôde ser aplicado às áreas pesquisadas.

\section{RESULTADOS E DISCUSSÃO}

Para o desenvolvimento da pesquisa foi analisado um total de 16 (dezesseis) periódicos eletrônicos, obtidos entre os periódicos levantados na pesquisa. A fórmula para detectar a pontuação do periódico eletrônico foi aplicada a grupos temáticos de periódicos selecionados (Tabela 1).

O resultado obtido na área da Ciência da Informação confirma a avaliação de relevância acerca dos periódicos analisados: Ciência da Informação é um periódico tradicional e consolidado na área, publicado pelo IBICT desde 1970 na versão impressa e desde 1998 em versão eletrônica. DatagramaZero é um novo e inovador periódico eletrônico, publicado desde 2001, que possui uma consistente política de divulgação entre os pesquisadores da área, e é editado por reconhecido pesquisador da área de Ciência da Informação (Tabela 1).

Finalmente, o periódico Informação \& Sociedade é um periódico acadêmico, editado pelo Programa de Pós-Graduação em Ciência da Informação da Universidade Federal da Paraíba. Não possui cobertura nacional, sendo considerado um periódico acadêmico de âmbito regional em virtude de seu escopo e de suas ligações com esse programa de pós-graduação. Desde o final de 2003 é editado apenas na versão eletrônica (Tabela 1).

O periódico que recebeu maior pontuação foi a Revista Eletrônica de Administração. Comparado com os outros dois analisados, recebeu mais links autoridades (sites de vários cursos de graduação e pós-graduação), o que representa um grau de maior significância, se comparado com os demais. Um especialista da área confirmou o resultado da pesquisa (Tabela 2).

Todos os periódicos analisados são arrolados no portal SciELO, significando que também possuem versão impressa. O resultado da avaliação coincide com a avaliação de especialista da área: Cadernos

Tabela 1. Área: Ciências Sociais Aplicadas - Ciência da Informação.

\begin{tabular}{|c|c|c|c|c|c|c|c|c|}
\hline $\begin{array}{l}\text { Periódico } \\
\text { eletrônico }\end{array}$ & $\begin{array}{l}\text { Incluído no } \\
\text { SciELO }\end{array}$ & $\begin{array}{l}\text { Total de } \\
\text { links }\end{array}$ & $\begin{array}{l}\text { Total de } \\
\text { links } \\
\text { repetidos }\end{array}$ & $\begin{array}{c}\text { Total de } \\
\text { links } \\
\text { SciELO }\end{array}$ & $\begin{array}{c}\text { Total de } \\
\text { links } \\
\text { estrangeiros }\end{array}$ & $\begin{array}{c}\text { Total de } \\
\text { links } \\
\text { autoridades }\end{array}$ & $\begin{array}{c}\text { Total de } \\
\text { links } \\
\text { simples }\end{array}$ & $\begin{array}{l}\text { Pontos } \\
\text { (G) }\end{array}$ \\
\hline Ciência da Informação & Sim & 43 & 0 & 0 & 7 & 6 & 30 & 62 \\
\hline Datagrama Zero & Não & 37 & 0 & 0 & 8 & 5 & 25 & 56 \\
\hline Informação \& Sociedade & Não & 18 & 0 & 0 & 5 & 5 & 8 & 33 \\
\hline
\end{tabular}

Tabela 2. Área: Ciências Sociais Aplicadas - Administração.

\begin{tabular}{|c|c|c|c|c|c|c|c|c|}
\hline $\begin{array}{l}\text { Periódico } \\
\text { eletrônico }\end{array}$ & $\begin{array}{l}\text { Incluído no } \\
\text { SciELO }\end{array}$ & $\begin{array}{l}\text { Total de } \\
\text { links }\end{array}$ & $\begin{array}{l}\text { Total de } \\
\text { links } \\
\text { repetidos }\end{array}$ & $\begin{array}{l}\text { Total de } \\
\text { links } \\
\text { SciELO }\end{array}$ & $\begin{array}{c}\text { Total de } \\
\text { links } \\
\text { estrangeiros }\end{array}$ & $\begin{array}{c}\text { Total de } \\
\text { links } \\
\text { autoridades }\end{array}$ & $\begin{array}{l}\text { Total de } \\
\text { links } \\
\text { simples }\end{array}$ & $\begin{array}{l}\text { Pontos } \\
\text { (G) }\end{array}$ \\
\hline Administração On-line & Não & 10 & 0 & 0 & 1 & 1 & 8 & 13 \\
\hline $\begin{array}{l}\text { Acadêmica-Revista Virtual } \\
\text { Revista Eletrônica de }\end{array}$ & Não & 5 & 0 & 0 & 2 & 0 & 3 & 7 \\
\hline Administração & Não & 36 & 16 & 0 & 2 & 6 & 12 & 34 \\
\hline
\end{tabular}


de Saúde Pública é o periódico mais relevante entre os analisados (Tabela 3).

O resultado obtido nessa área causou uma surpresa. Dentre os dois periódicos eletrônicos analisados, a Revista Latino-Americana de Enfermagem, incluída no portal SciELO, recebeu uma pontuação bem menor da recebida pelo periódico On-line Brazilian Journal of Nursing, não incluído no SciELO. Acredita-se que isso tenha ocorrido pelo fato de o referido periódico ser publicado na língua inglesa, o que o torna mais acessível à comunidade interna- cional, fato comprovado pelo total de links estrangeiros apurado na pesquisa. No entanto, não houve confirmação de especialista da área quanto ao resultado (Tabela 4).

Todos os periódicos analisados pertencem ao portal SciELO. Esse grupo de periódicos eletrônicos recebeu poucos links. Para avaliá los contou-se com o auxílio de especialista da área que, no entanto, não confirmou o resultado obtido por meio da aplicação do método de avaliação proposta, indicando o periódico Brazilian Journal of Veterinary

Tabela 3. Área: Ciências da Saúde - Saúde Pública.

\begin{tabular}{|c|c|c|c|c|c|c|c|c|}
\hline $\begin{array}{l}\text { Periódico } \\
\text { eletrônico }\end{array}$ & $\begin{array}{l}\text { Incluído no } \\
\text { SciELO }\end{array}$ & $\begin{array}{c}\text { Total de } \\
\text { links }\end{array}$ & $\begin{array}{c}\text { Total de } \\
\text { links } \\
\text { repetidos }\end{array}$ & $\begin{array}{l}\text { Total de } \\
\text { links } \\
\text { SciELO }\end{array}$ & $\begin{array}{c}\text { Total de } \\
\text { links } \\
\text { estrangeiros }\end{array}$ & $\begin{array}{c}\text { Total de } \\
\text { links } \\
\text { autoridades }\end{array}$ & $\begin{array}{l}\text { Total de } \\
\text { links } \\
\text { simples }\end{array}$ & $\begin{array}{c}\text { Pontos } \\
\text { (G) }\end{array}$ \\
\hline Cadernos de Saúde Pública & Sim & 100 & 0 & 87 & 10 & 2 & 1 & 27 \\
\hline Revista de Saúde Pública & Sim & 100 & 0 & 94 & 6 & 0 & 0 & 12 \\
\hline Revista de Saúde Coletiva & Sim & 62 & 0 & 57 & 3 & 0 & 2 & 8 \\
\hline
\end{tabular}

Tabela 4. Área: Ciências da Saúde-Enfermagem.

\begin{tabular}{|c|c|c|c|c|c|c|c|c|}
\hline $\begin{array}{l}\text { Periódico } \\
\text { eletrônico }\end{array}$ & $\begin{array}{l}\text { Incluído no } \\
\text { SciELO }\end{array}$ & $\begin{array}{l}\text { Total de } \\
\text { Links }\end{array}$ & $\begin{array}{l}\text { Total de } \\
\text { links } \\
\text { repetidos }\end{array}$ & $\begin{array}{l}\text { Total de } \\
\text { links } \\
\text { SciELO }\end{array}$ & $\begin{array}{c}\text { Total de } \\
\text { links } \\
\text { estrangeiros }\end{array}$ & $\begin{array}{c}\text { Total de } \\
\text { links } \\
\text { autoridades }\end{array}$ & $\begin{array}{l}\text { Total de } \\
\text { links } \\
\text { simples }\end{array}$ & $\begin{array}{l}\text { Pontos } \\
\text { (G) }\end{array}$ \\
\hline $\begin{array}{l}\text { On-line Brazilian Journal of } \\
\text { Nursing }\end{array}$ & Não & 29 & 0 & 0 & 27 & 1 & 1 & 58 \\
\hline $\begin{array}{l}\text { Revista Latino- Americana de } \\
\text { Enfermagem }\end{array}$ & Não & 75 & 0 & 68 & 4 & 0 & 3 & 11 \\
\hline
\end{tabular}

Tabela 5. Área: Ciências Agrárias - Veterinária.

\begin{tabular}{|c|c|c|c|c|c|c|c|c|}
\hline $\begin{array}{l}\text { Periódico } \\
\text { eletrônico }\end{array}$ & $\begin{array}{l}\text { Incluído no } \\
\text { SciELO }\end{array}$ & $\begin{array}{l}\text { Total de } \\
\text { links }\end{array}$ & $\begin{array}{l}\text { Total de } \\
\text { links } \\
\text { repetidos }\end{array}$ & $\begin{array}{c}\text { Total de } \\
\text { links } \\
\text { SciELO }\end{array}$ & $\begin{array}{c}\text { Total de } \\
\text { links } \\
\text { estrangeiros }\end{array}$ & $\begin{array}{c}\text { Total de } \\
\text { links } \\
\text { autoridades }\end{array}$ & $\begin{array}{l}\text { Total de } \\
\text { links } \\
\text { simples }\end{array}$ & $\begin{array}{l}\text { Pontos } \\
\text { (G) }\end{array}$ \\
\hline $\begin{array}{l}\text { Arquivos Brasileiros de Medicina } \\
\text { VeterináriaeZootecnia }\end{array}$ & Sim & 25 & 0 & 10 & 9 & 1 & 5 & 26 \\
\hline Pesquisa VeterináriaBrasileira & Sim & 53 & 0 & 42 & 6 & 1 & 4 & 19 \\
\hline $\begin{array}{l}\text { Brazilian Journal of Veterinary } \\
\text { Research and Animal Science }\end{array}$ & Sim & 22 & 0 & 14 & 4 & 1 & 3 & 15 \\
\hline
\end{tabular}

Tabela 6. Área: Humanidades - Educação.

\begin{tabular}{|c|c|c|c|c|c|c|c|c|}
\hline $\begin{array}{l}\text { Periódico } \\
\text { eletrônico }\end{array}$ & $\begin{array}{l}\text { Incluído no } \\
\text { SciELO }\end{array}$ & $\begin{array}{c}\text { Total de } \\
\text { links }\end{array}$ & $\begin{array}{l}\text { Total de } \\
\text { links } \\
\text { repetidos }\end{array}$ & $\begin{array}{c}\text { Total de } \\
\text { links } \\
\text { SciELO }\end{array}$ & $\begin{array}{c}\text { Total de } \\
\text { links } \\
\text { estrangeiros }\end{array}$ & $\begin{array}{c}\text { Total de } \\
\text { links } \\
\text { autoridades }\end{array}$ & $\begin{array}{l}\text { Total de } \\
\text { links } \\
\text { simples }\end{array}$ & $\begin{array}{l}\text { Pontos } \\
\text { (G) }\end{array}$ \\
\hline EducaçãoePesquisa & Sim & 67 & 0 & 60 & 7 & 0 & 0 & 14 \\
\hline Educação\& Sociedade & Sim & 11 & 0 & 2 & 6 & 0 & 3 & 15 \\
\hline
\end{tabular}


Research and Animal Science como o mais importante periódico eletrônico dentre os três analisados (Tabela 5).

Os dois periódicos eletrônicos analisados pertencem ao portal SciELO. O resultado da pesquisa apontou os dois periódicos com praticamente o mesmo grau de relevância. No entanto, especialista da área indicou o periódico Educação e Sociedade como sendo o mais importante (Tabela 6 ).

\section{O N CLUS Ã O}

De maneira geral, o grupo de pesquisa sentiu certa dificuldade em aplicar a fórmula desenvolvida para aferir graus indicativos de relevância em periódicos eletrônicos brasileiros de áreas que não pertencem ao seu domínio de conhecimento, como é o caso da área da Ciência da Informação. Por outro lado, os periódicos científicos em formato eletrônico ditos emergentes representam uma realidade não completamente familiar à maioria da comunidade acadêmica brasileira. Conforme Brown, citado por Hyldegaard e Seiden (2004), "as características das áreas científicas afetaram a preferência de cientistas por fontes de informação", o que tem dificultado encontrar parceiros na comunidade de pesquisa que conheçam os periódicos eletrônicos emergentes selecionados e submetidos à fórmula proposta e que possam validar o resultado da pesquisa.

Foi também difícil encontrar, no universo dos periódicos eletrônicos brasileiros, áreas do conhecimento que possuíssem mais de um periódico, caso específico da Física (Brazilian Journal of Physics). Um pesquisador da área informou que essa comunidade científica prefere publicar em periódicos internacionais e, se possível, em periódicos impressos.

A mesma situação foi encontrada na área de Ciência da Computação. Esses tipos de problemas dificultaram a aplicação do método em algumas áreas do conhecimento. Assim, considera-se como ideal para avaliar o método proposto, a existência de mais de um periódico eletrônico na área de conhecimento a ser analisada e a assistência de pesquisadores da área, conhecedores dos periódicos analisados, para ratificar o resultado encontrado pela aplicação da fórmula.

Embora os resultados obtidos por meio da aplicação da fórmula coincidam com a avaliação de relevância dos periódicos eletrônicos na área de Ciência da Informação, o grupo considerou a área atípica, talvez pelo fato de, na prática, os profissionais atuantes em bibliotecas e em faculdades de biblioteconomia brasileiras contarem com bons serviços de referência eletrônica. Há que se considerar também que os três periódicos eletrônicos, juntamente com outros três existentes na área, são recomendados pelas principais escolas de biblioteconomia e bibliotecas acadêmicas brasileiras que possuem em suas home pages informações sobre cursos de graduação e pós-graduação em Biblioteconomia e Ciência da Informação. O conceito de autoridade aplicado a sites também se mostrou satisfatório na área.

Considera-se também como hipótese a de sites de programas de pós-graduação em diferentes áreas serem considerados como autoridade. Isso foi verificado em poucas áreas, como, por exemplo, na Administração. Entretanto, em muitos sites de cursos de graduação foram encontrados links para periódicos eletrônicos em diferentes áreas, sendo considerados também como autoridades.

Constatou-se também que, com exceção da área de Ciência da Informação e da área de Ciências da Saúde, mais precisamente na Universidade Federal de São Paulo, importante centro de pós-graduação e pesquisa em ciências da saúde, parece haver poucos serviços de referência eletrônica nas bibliotecas acadêmicas e universitárias, ou mesmo de centros de pesquisa brasileiros. E, mesmo quando existentes, não incluem os periódicos eletrônicos brasileiros, o que evidencia a importância dada aos periódicos estrangeiros pela comunidade de pesquisa brasileira em detrimento dos nacionais.

Acredita-se, após esses primeiros resultados obtidos, ser necessário definir quais são as melhores condições de aplicação da fórmula desenvolvida. $O$ conceito de autoridade usado deve ser ampliado, talvez incluindo autoridades estrangeiras. É necessário, também, contar com o apoio mais consistente de pesquisadores das áreas específicas dos periódicos eletrônicos analisados. É preciso estabelecer uma forma de automatizar esse método, após serem feitas as devidas correções, para que ele possa ser publicamente avaliado em um site da WEB. Acredita-se ainda que a aplicação de forma sistemática e segura do método proposto dependa muito da implementação de um serviço de referência eletrônica pelas bibliotecas universitárias brasileiras. 
A avaliação da qualidade é essencial para consolidar os periódicos eletrônicos brasileiros em C \& T ditos emergentes junto à comunidade científica brasileira, e um sistema de avaliação permanente é necessário para impingir esse conceito de qualidade. Esses periódicos encontram dificuldades em satisfazer os critérios de qualidade estabelecidos pelo fator de impacto ISI, por isso acredita-se ser necessária a adoção de métodos alternativos e de políticas públicas que contemplem esse tema.

O uso da fórmula proposta para aferir graus de relevância aos periódicos eletrônicos, como dito anteriormente, precisa ser mais do que um simples método. Poderia ser adotado na forma de política pública, juntamente com outras políticas envolvendo

\section{REFERÊNCIAS}

DAY, M. The scholarly journal in transition and the PubMed Central proposal. Ariadne, v.21, Sept, 1999. Available from: <http://www.ariadne.ac.uk/issue21/pubmed/>. Acess: 15 Aug. 2004.

GARFIELD, E. The Impact factor. Current Contents, June, 1994. Available from: <http://www.isinet.com/isi/hot/essays/ journalcitationreports/7.html>. Acess: 18 Aug. 2004.

GIBBS, W.W. Lost science in the Third World. Scientific American, v.273, n.2, p.76-83, 1995.

GINSPARG, P. Winners and losers in the global research village. In: CONFERENCE ON ELECTRONIC PUBLISHING IN SCIENCE, 1996, Paris. Proceedings... Available from: <http:/ /xxx.lanl.gov/blurb/pg96unesco.html>. Acess: 5 Oct. 2001.

HYLDEGAARD, J.; SEIDEN, P. My e-journal: exploring the usefulness of personalized access to scholarly articles and services. Information Research, v.9, n.3, 2004. Available from: $<$ http://information.net/ir/9-3/paper181.html>. Acess: 8 Nov. 2004.

KLEINBERG, J.M. Authoritative Sources in a hyperlinked environment. In: ANNUAL ACM-SIAM SYMPOSIUM ON DISCRETE ALGORITHMS, 9th, San Francisco, California. Proceedings... New York: ACM PRESS, p.668-677. 1998. Available from: <http://doi.acm.org/10.1145/314613.315045>. Acess: 12 Jun. 2004.

KLEINBERG, J.M.; LAWRENCE, S. The structure of the Web. Science, v.294, p.1849-1850, 2001.

LAWRENCE, S. Online or invisible? Nature, v.411, n.6837, p.521, 2001. Available from: <http://www.nature.com/nature/ debates/e-access/Articles/lawrence.html>. Acess: 10 Jun. 2001. MARCONDES, C.H.; SAYÃO, L.F.; MAIA, C.M.; DANTAS, M.A.R., FARIA, W.S. State of the art of Brazilian ejournals in science and technology. In: INTERNATIONAL CONFERENCE ON ELECTRONIC PUBLISHING, EIPub, 8th Brasília, 2004, Proceedings... Brasília, 2004. Available from: <http:// elpub.scix.net>. Acess: 7 Feb. 2005.

MEADOWS, A.J. A comunicação científica. Brasília: Briquet de Lemos, 1999. periódicos eletrônicos, como acontece nos portais do SciELO e da Capes. As comunidades de pesquisa e bibliotecárias pertencentes a uma área do conhecimento precisam concordar com o grupo de instituições consideradas autoridades. Um site da WEB poderia ser desenvolvido com os links acordados pela comunidade como autoridades. Dessa forma, um editor pode submeter a este Website o link do seu periódico eletrônico; a fórmula seria nele aplicada e a pontuação obtida ficaria disponível na WEB para toda a comunidade de pesquisa. Um site como o descrito poderia ser um importante instrumento para avaliação dos emergentes periódicos eletrônicos acadêmicos brasileiros.
ODLYSKO, A. The economic of electronic journals. Journal of Electronic Publishing, v.4, n.1, Sept. 1998. Available from: <http://www.press.umich.edu/jep/04-01/odlyzko.html>. Acess: 13 Sept. 2004.

PACKER, A.L. SciELO: a Model for Cooperative Electronic Publishing in Developing Countries. D-Lib Magazine [online], v.6, n.10, 2000. Available from: <http://www.dlib.org/dlib/ october00/10inbrief.html\#PACKER>. Acess: 15 Sept. 2004.

PACKER,A.L. The SciELO Model for electronic publishing and measuring of usage and impact of Latin American and Caribbean scientific journals. In ICSU-UNESCO International Conference: Electronic Publishing in Science, 2, Paris, 2001, Proceedings. [online]. Paris, 2001, p.19-23. Available from: <http://www.unesco.org/science/publication/electronic _publishing_2001/proceedings_sess3.shtml\#s3_packer>. Acess: 27 Aug. 2004.

SNYDER, H.; ROSENBAUN, H. Can search engines be used as tools for web-link analysis?: a critical view. Journal of Documentation, v.55, n.4, p.375-384. 1999. Available from: <http://www.periodicos.capes.gov.br>. Acess: 2 Dec. 2004.

TARGINO, M.G.; GARCIA, J.C.R. Ciência brasileira na base de dados do Institute for Scientific Information. Ciência da Informação, Brasília, v.29, n.1, p.103-117, jan./abr. 2000. Disponível em: <http://www.scielo.br/scielo.php?script= sci_arttext\&pid=S0100-19652000000100011\&lng=pt \&nrm=iso>. Acesso em: 14 fev. 2005.

TESTA, J. The ISI database: the journal selection process. 1997. Available from: <http://www.isinet.com/essaysselectionof materialforcoverage/199701.html>. Acess: 21 Sept. 2004.

VAUGHAN, L., HYSEN, K. Relationship between links to journal Web sites and impact factors. ASLIB Proceeedings, v.54, n.6, p.356-361, 2002. Available from: <http://mismain. bsa.kent. edu/Users/weinroth/public/jing2.pdf>. Acess: 14 Nov. 2004.

VAUGHAN, L.; THEWALL, M. Scholarly use of Web: what are the key inducers of links to journal Web sites? Journal of The American Society for Information Science and Technology, v.54 n.1, p.29-38, 2003. Available from: <http://www.periodicos. capes.gov.br>. Acess: 2 Dec. 2004. 